EPJ Web of Conferences 23, 00007 (2012)

DOI: $10.1051 /$ epjconf/20122300007

(C) Owned by the authors, published by EDP Sciences, 2012

\title{
Phenomenological Theory for Pseudogap States in High $\mathbf{T}_{c}$ Cuprate
}

Fuchun Zhang

Department of Physics, The University of Hong Kong, Hong Kong, China

\begin{abstract}
Pseudogap phase in the underdoped region of high-Tc cuprate is one of the challenging issues in condensed matter physics. In this talk, I will describe a phenomenological theory for this phase, based on analogies to the approach to Mott localization at weak coupling in lower dimensional systems. I will make comparisons of the theory to a series of the experiments, including angle resolved photoemission spectroscope, scanning tunneling microscope.
\end{abstract}

This is an Open Access article distributed under the terms of the Creative Commons Attribution-Noncommercial License 3.0, which permits unrestricted use, distribution, and reproduction in any noncommercial medium, provided the original work is properly cited. 